\title{
Psychometric Testing of The Chinese Version of The Family Crisis Oriented Personal Evaluation Scales In Families of Patients With Dementia
}

\section{Qingyan Wang}

Xuzhou Medical University

\section{Sanmei Chen}

Hiroshima University

Weiwei Liu

Army Medical University

Yu Sheng ( $\square$ shengyum@126.com )

Peking Union Medical College

\section{Research Article}

Keywords: Family characteristics, Asian continental ancestry group, Behaviour rating scale, Dementia

Posted Date: September 24th, 2021

DOI: https://doi.org/10.21203/rs.3.rs-840922/v1

License: (c) (i) This work is licensed under a Creative Commons Attribution 4.0 International License. Read Full License 


\section{Abstract}

Background: The Family Crisis Oriented Personal Evaluation Scales (F-COPES) is a widely used instrument to evaluate family coping behaviours. However, no Chinese version of this scale have been developed and validated in China. This study aimed to develop a Chinese version of the F-COPES and evaluate its psychometric properties in the families of patients with dementia who have heavy stress and care burdens.

Methods: A cross-sectional study in the specialist memory and geriatric psychiatric clinics of four hospitals in Beijing, China. The English version of the F-COPES was translated into Chinese according to the standard translation guideline. An expert panel was invited to evaluate the content validity index of the Chinese version of this scale. This study included 215 families of patients with dementia. The item homogeneity analysis was conducted by using corrected item-total correlation, corrected item-subscale correlation, and changes of Cronbach's alpha coefficient of subscales after deleting each item. The factor structure was analysed by using a confirmatory factor analysis. The convergent validity was tested by correlating the F-COPES to the Multidimensional Scale of Perceived Social Support (MSPSS). The internal consistency was assessed by using Cronbach's alpha coefficients of the whole scale and subscales.

Results: The content validity index of the Chinese version of the F-COPES was 0.98 , with that of each item ranging from 0.83 to 1.00 . The result of item homogeneity analysis was satisfactory except for the items in the subscale of passive appraisal. The confirmatory factor analysis identified six subscales; namely, acquiring relatives' support, acquiring friends' support, acquiring neighbours' or others' support, reframing, seeking spiritual support, and passive appraisal, with acceptable model fit indices $\left(X^{2} / d f=1.65, C F I=\right.$ $0.91, \mathrm{GFI}=0.85, \mathrm{TLI}=0.90, \mathrm{PGFI}=0.69, \mathrm{RMSEA}=0.06)$. The convergent correlation between the F-COPES and the MSPSS was strong $(r=0.50, P<0.01)$. The Cronbach's alpha coefficients of the whole scale was 0.86 .

Conclusion: The Chinese version of the F-COPES showed satisfactory psychometric properties. It may serve as a useful scale for assessing the coping behaviours in families of patients with dementia in China.

\section{Introduction}

Dementia is a chronic and progressive syndrome, caused by a variety of brain illnesses affecting memory, thinking, behaviours, and abilities to perform activities of daily living [1]. People with moderate dementia can no longer live independently, and people with severe dementia rely almost entirely on others' caregiving [2]. The number of people living with dementia worldwide is currently estimated at 47 million, expected to increase to 82 million by 2030 [2]. The majority ( $80 \%$ ) of people with dementia are receiving care in their homes [3], which leads their families, especially the families of patients with moderate to severe dementia, to have impaired physical and psychological health as a result [4]. The families included the spouses, children, children-in-law, and grandchildren who were living together with the patients or 
taking care of the patients on a routine basis [5]. The patients' dependence on aspects of daily living causes heavy stress and care burdens to their families [6]. Coping of families refers to specific efforts that an individual family member or the family functioning as a whole makes to reduce the stress and burden, such as managing the demand on the family system or utilizing coping resources [7]. Coping ability is important in decreasing such stress and promoting family adaptation [8,9]. A tool to identify the coping abilities of families of patients with dementia is necessary to identify vulnerable families with insufficient coping abilities and the coping abilities which they lack, which helps develop interventions to enhance the coping ability of these families.

The Family Crisis Oriented Personal Evaluation Scales (F-COPES) was the most commonly used instrument to measure family coping via self-reporting of family members of medically fragile adults [10]. McCubbin and his colleagues developed and validated the original English version of this scale based on their Resiliency Model, which integrated both internal and external factors influencing coping abilities of a family (i.e., family resources, social support, and cognition) [7]. In the resilience model, family coping abilities were defined as the following two levels of efforts: (1) the efforts the family makes to handle difficulties and problems via internal resources or cognition inside the family, and (2) the efforts that the family makes to handle problems or demands via externally social support outside family boundaries [7]. The original English version included 30 items and five subscales, including the subscale of acquiring social support, the subscale of reframing, the subscale of seeking spiritual support, the subscale of mobilizing the family to acquire and accept help, and the subscale of passive appraisal [7]. The original English version has been translated into different languages, resulting in the Persian version, Malay version, and Turkish version, etc. [10-12]. Two small studies of Chinese-American and Hong Kong Chinese established Chinese versions of the F-COPES among breast cancer survivors and critically ill patients, respectively $[13,14]$. However, those Chinese versions cannot be directly used in mainland Chinese populations, due to the distinct social and cultural environments in China [15]. To the best of our knowledge, to date, there are no Chinese versions of this scale developed and validated in mainland China. Thus, in the present study, we developed a Chinese version of the F-COPES using the standard procedure of translation and cultural adaptation, and evaluated its psychometric properties in families of patients with dementia in mainland China.

\section{Methods}

\section{Setting and sample}

The study population was comprised of family members of patients with dementia who visited the specialist memory and geriatric psychiatric clinics of four hospitals in Beijing of China from October 2017 to March 2018. Family members were considered eligible for this study if their relatives with dementia were taken care of at home for at least six months. According to the equations of the minimum sample size $\left(\mathrm{N}_{\epsilon}=\frac{\delta_{1-\beta}}{\epsilon^{2} \mathrm{df}}+1\right)$, the minimum sample size of our study was 108 , when the $\mathrm{df}=218, \varepsilon=0.05$, the power $(1-\beta)=0.80$, and the $\delta_{1-\beta}=58.182$ ( $\delta$ is the non-centrality parameter ) [16]. We initially recruited 
250 patients who were diagnosed with dementia at least six months prior. Through the physician's referral, we invited one to two family members of each patient, who were the primary caregivers of each patient to fulfil our scales on behalf of the family. In total, 250 family members were invited to participate in our survey. Of those, 25 refused to participate in this study because they had no interest or no time to answer the questions. Thus, a total of 225 family members agreed to participate in this study. For this analysis, we excluded 10 participants who had data missing for more than $10 \%$ of the survey. Therefore, the final sample included 215 participants (Fig. 1). The Ethical Review Committee of School of Nursing, Peking Union Medical College approved the study protocol in April 15 (Approval no. [2015] 03) and the study was conducted in accordance with the principles of the Declaration of Helsinki. All of the participants were informed about the aim and significance of this study, their right to quit at any time, and how to fill in the scales. All participants provided written informed consent.

\section{Measurements}

\section{Characteristics of patients and families}

Information on the following variates was collected through the questionnaire, including demographic and disease-related characteristics of patients, and demographic characteristics of family members as well as the whole family. The patient information included age, gender, subtypes of dementia (Alzheimer's disease, vascular dementia, and others or unknown), dementia severity based on Clinical Dementia Rating evaluated by the physician (moderate, severe), and time or duration since diagnosis. The information on family members and the whole family included age, educational level (junior middle school and below, high school, college degree, and graduate degree), whether they have religious faith (yes or no), and family income per month per person.

\section{Family Crisis Oriented Personal Evaluation Scales (F-COPES)}

The F-COPES was the most commonly used instrument to measure family coping via self-report. It was comprised of 30 item and five subscales. The five subscales included a subscale of acquiring social support (the family's behaviours of actively engaging in acquiring support from extended family, friends, and neighbours), subscale of mobilizing the family to acquire and accept help (the family's behaviours of seeking out and using community resources to cope with problems), subscale of seeking spiritual support (the family's behaviours or attitudes of acquiring spiritual support), subscale of reframing (the family's behaviours or attitudes of redefining stresses/situations to make them more manageable), and subscale of passive appraisal (passive/inactive coping behaviours or attitudes in managing problems). Respondents were asked to choose the frequency of using different coping methods on a scale of 1 (almost never) to 5 (almost always). Items of Nos. 12, 17, 26, and 28 should be reverse scored when obtaining the total score of the F-COPES by summing the responses of all items. This is due to the fact that the coping behaviours or attitudes described in the items of Nos. 12, 17, 26, and 28 were considered negative. Higher total scores of the F-COPES represent better family coping with stressful situations. The 
F-COPES has strong internal consistency with Cronbach's alpha coefficient ranging from 0.61 to 0.87 [17, $18]$, and good factorial and concurrent validities $[19,20]$.

\section{Multidimensional Scale of Perceived Social Support (MSPSS)}

The MSPSS was selected to evaluate the convergent validity of F-COPES in this study. The MSPSS measured the perceived social support from three informal sources: family, friends, and significant other. Participants were rated on a seven-point Likert response format $(1=$ "very strongly disagree" to 7 = "very strongly agree"). The total score is summed by the scores of items, with higher scores indicative of greater perceived social support. Zimet et al. [21] tested the MSPSS and reported high internal consistency of 0.88 . The test-retest reliability was 0.85 over a 2 - to 3 -months period after completing the questionnaire [21]. The MSPSS had a Cronbach's alpha coefficient of 0.90 in the current study.

\section{Procedure}

\section{Translation and adaptation of F-COPES}

We conducted a rigorous "forward-backward" translation following the guidelines to develop the initial Chinese version of F-COPES [22]. Then, during the adaptation process, we invited six clinical nurse supervisors, nursing researchers, or psychological researchers who were professors or associate professors and familiar with both dementia caregiving and psychometrics to form an expert panel to judge whether each item of the initial Chinese version of F-COPES should be deleted or modified and whether we should add other items.

In the adaptation process, we deleted the items of Nos. 9, 29, and 30 from the Chinese version of the FCOPES. Item 9 ("Seeking information and advice from the family doctor") was deleted because the system of family doctors, which is commonly understood internationally, does not exist in China [23]. Item 29 ("Sharing problems with neighbours") was deleted because families of patients with dementia are reluctant to share problems with neighbours when facing the discrimination and prejudice against patients with dementia and their families in China [24, 25]. Item 30 ("Having faith in God") was deleted, because only $10.4 \%$ of Chinese participants identified themselves as religious [26]. Only $6.5 \%$ of family members in the current study had religious faith, although they participate in religious practices. We modified the items (items of Nos. 14,23,27) that described religious practices as coping methods in the subscale of seeking spiritual support. We broadened the Christian terms in these items to comprehensively suit other religions, which was similar to the Turkish version of the F-COPES [12] since the Chinese participants were engaged in some form of religious practice across the various religions [26]. Finally, all experts agreed there was no need to add any new items.

\section{Statistical analysis}

After translation and adaptation of the F-COPES, we did a content validity analysis, item homogeneity analysis, factor structure analysis, convergent validity analysis, and internal consistency reliability of the 
F-COPES. We used AMOS 20.0 software for factor structure analysis and SPSS 17.0 software for other analyses.

Content validity analysis was explored by the item content validity index (I-CVI) and the scale content validity (S-CVI). Six experts were invited to quantify the relevance of items to family coping by using a rating scale (not relevant or somewhat relevant $=0$, quite relevant, and very relevant $=1$ ). The $I-C V I$ is the average of all experts rating the results of each item, and S-CVI is the average of I-CVIs. The I-CVI is recommended to be no lower than 0.78 and an S-CVI of 0.80 or higher is acceptable $[27,28]$. Item homogeneity analysis was tested using a corrected item-subscale correlation, item-total correlation, and Cronbach's alpha coefficient of the subscale after deleting each item. The corresponding item is acceptable if the corrected item-subscale correlation is higher than 0.20 , and the value of Cronbach's alpha coefficient of the scale after deleting each item is lower than before the deletion [29].

The factor structure of the F-COPES was analysed by confirmatory factor analysis. The criteria used to determine whether the models of factor structure fit the data were 3.00 or lower on the chi-square of model fit/degree of freedom ( $\left.\chi^{2} / d f\right)$ [30], 0.90 or greater on the comparative goodness-of-fit index (CFI), goodness-of-fit index (GFI), and the Tucker-Lewis Index (TLI); 0.50 or greater on parsimony goodness-of-fit index (PGFI) [31-34]; and 0.08 or lower on a root-mean-square error of approximation (RMSEA) [35]. In the confirmatory factor analysis in the current study, the original model established based on the factor structure of the English version of the F-COPES was tested first. The competing model was then tested if the original model did not meet the above-mentioned criteria [36]. The data fitness of the original model and competing models of F-COPES were compared. If the competing model -which meets the above fitness criteria and the value of CFI of this model-is at least 0.01 higher than the original model, the competing model is then considered significantly better than the original one [37]. Convergent validity of the F-COPES was tested by estimating its correlation with social supports as measured by the MSPSS, since social support has been shown be to be correlated to caregiver coping [38]. The correlation coefficient for convergent validity between 0.10 and 0.29 was considered weak, 0.30 to 0.49 was considered moderate, and 0.50 to 1.0 was considered strong [39]. The internal consistency reliability of the scale and subscales were evaluated by Cronbach's alpha coefficients. A Cronbach's alpha coefficient between 0.80 and 0.90 was considered perfect, and 0.65 was the lowest acceptable cut point [40].

\section{Results}

\section{Demographics}

Table 1 shows the characteristics of patients and families. Among 215 families, the median age of patients was 82.0 (interquartile range: $76.0-86.0$ ) years old, and $65.1 \%$ were women. Alzheimer's disease accounted for $57.2 \%$. The median age of family members were $59.5(53.3-65.0)$ years old, and $60.4 \%$ 
had an educational level of a college degree or above. In these families, only $6.5 \%$ of the family members had religious faith.

\section{Content validity}

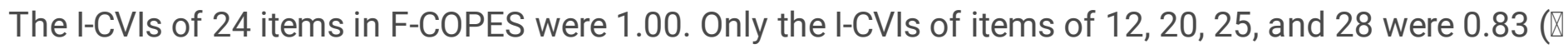
0.78 is acceptable). The S-CVI was equal to 0.98 by calculating the average of the I-CVls of all items ( $\mathbb{Z}$ 0.80 is acceptable).

\section{Item homogeneity}

Table 2 shows the results of item homogeneity of each item and the whole scale. The results of item homogeneity tested by the corrected item-subscale correlation were above 0.20 (ranged from 0.28 to 0.84 ), except for items in the subscale of original passive appraisal (ranged from -0.01 to 0.09). The Cronbach's alpha coefficient of subscales after deleting each item was lower than before, excepts for items in the subscale of original passive appraisal. The results of Cronbach's alpha coefficient had also noticed that the unsatisfactory results of the subscale of passive appraisal may be mistakes in scoring the items in this subscale. According to the negative corrected item-total correlation of items of 12 and 28 in the passive appraisal subscale $(-0.26$ and -0.50 , respectively), we reversed the scoring method of item 12 and item 28 based on the above results and literature review. The corrected item-total correlation of item 12 and item 28 were positive in the modified subscale of paasive appraisal. The results of the corrected item-subscale correlation and Cronbach's alpha coefficient of the subscale after deleting each item were improved.

\section{Factor structure}

Table 3 shows the fit statistics for the original factor structure and the competing structure of the Chinese version of the F-COPES in the present study. The values of CFI, GFI, and TLI of the original model did not meet the criteria. We, therefore, established a six-factor competing model based on parts of the original model and the classification of social support [41]. In the competing model, we put the following 12 items together: three items in the original subscale of mobilizing the family to acquire and accept help, and nine items in the original subscale of acquiring social support. Then, we divided these 12 items into three new subscales depending on the source of the social support; namely, acquiring relatives'support, acquiring friends' support, and acquiring neighbours'or others' support. The values of CFI and GFI of the competing model met the criteria. Additionally, the value of CFI of the competing model is 0.05 higher than that of the original model, indicating the competing model was significantly better than the original one. Figure 2 shows the factor structure of the F-COPES identified based on the competing model, including six factor, the correlations between the six factors, the factor-loading from the six factors of the F-COPES to each item, the square of the factor-loading of each item, and the correlation between the errors of items based on standardized estimates. 
In the competing model, we deleted the correlations between the subscale of seeking spiritual support and the other three subscales of reframing, passive appraisal, and acquiring relatives' support, because the correlations between them were not significant ( $P>0.05)$. We added the correlations between item 2 and item 16, between item 3 and item 7, between item 4 and item 20, between item 6 and item 10, between item 7 and item 22, and between item 8 and item 10 .

\section{Convergent validity}

Table 4 shows the correlation coefficients of each subscale score and the total score of the the Chinese version of the F-COPES with the total score of the MSPSS. The total score of F-COPES had a significantly strong correlation with the total score of the MSPSS $(r=0.50, P<0.01)$. The six subscales of the FCOPES significantly correlated with the total score of the MSPSS, except for the subscale of seeking spiritual support.

\section{Internal consistency reliability}

The Cronbach's alpha coefficient of this measure was 0.86 . The Cronbach's alpha coefficient of subscales based on the competing factor structure was 0.76 for the subscale of acquiring relatives' support, 0.66 for the subscale of acquiring friends' support, 0.68 for the subscale of acquiring neighbours' or others' support, 0.85 for the subscale of reframing, 0.90 for the subscale of seeking spiritual support, and 0.33 for the subscale of passive appraisal.

\section{Discussion}

In the present study in families of patients with dementia in mainland China, we developed a Chinese version of the F-COPES based on the English version and evaluated its psychometric properties. Our Chinese version of this scale showed satisfactory psychometric properties, including content validity, item homogeneity, factor structure, convergent validity, and internal consistency reliability of the whole scale. We also established the six-factor structure of the F-COPES, which is different from other language versions. The F-COPES may serve as a useful scale for assessing the coping behaviours in families of patients with dementia in China. The present study is one of few to develop and validate a Chinese version of the F-COPES in mainland China.

\section{Content validity}

The Chinese version of the F-COPES satisfactorily covered relevant domains of family coping, because the relevance of the items (i.e., content validity indexes of the whole scale and items) was perfect. The entire expert panel agreed that the included items in the Chinese version adequately represented the content or behaviours in the domain of family coping. Our findings are in line with those from several studies of different language versions (i.e., the Persian version and Turkish version ) which reported that the items in the F-COPES were representative internationally $[10,12]$. This could possibly owe to the 
integrated and systematic theoretical framework of the F-COPES based on the Resiliency Model developed and validated by McCubbin and his colleagues [6].

\section{Item homogeneity}

We found that the items in the subscales for acquiring social support, reframing, seeking spiritual support, and mobilizing the family to acquire and accept help were homogeneous, as indicated by the results of the corrected item-subscale correlation and Cronbach's alpha coefficient of the subscale after deleting each item. These findings were similar to those from other studies in different countries $[10,11]$.

Of note, the homogeneity of items in the subscales of passive appraisal was unsatisfactory because of the inappropriate original scoring of items 12 and 28 in the Chinese version. In the present study, the homogeneity of items in the passive appraisal subscale was improved after reversing into positive scoring for item 12-"watching TV"-and item 28-"believing if we wait long enough, the problem will go away." Item 12 in our Chinese version of the F-COPES should be positively scored because watching TV has proven to be a very helpful coping behaviour when dealing with high levels of stress [42, 43]. We also positively scored item 28 , which could be partly supported by the notion that "believing if we wait long enough, the problem will go away" may play a beneficial role in coping mechanisms for Chinese families when facing difficulties, because Chinese culture values the virtues of patience, waiting, and forbearance [13].

\section{Factor structure}

The subcomponents of family coping that the Chinese version of the F-COPES identifies in families of patients with dementia included acquiring relatives' support, acquiring friends' support, acquiring neighbours' or others' support, reframing, seeking spiritual support, and passive appraisal (Figure 2). This six-factor model of the Chinese version of F-COPES fit better the observed data as the results show in Table 3. This model was partly different from other language versions, such as the three-factor Turkish version [12] and the five-factor African American versions [44]. In the Chinese version, the 12 items in the original subscale of mobilizing the family to acquire and accept help and the subscale of acquiring social support were divided into three new subscales depending on the source of the social support. It supported the suggestion of a previous study, which encourages dividing the coping skills of "acquiring social support" depending on the source of the social support in the Chinese population because of its familism culture $[13,45,46]$.

Besides that, in the Chinese version of F-COPES, the correlations between the subscale of seeking spiritual support and the three subscales-reframing, passive appraisal, and acquiring relatives' supportdisappeared. There were not the same as results of other studies [13,44], perhaps related to different spiritual supporting environments and experiences. Only $6.5 \%$ of family members have religious faith in our study, and this is much fewer than other populations [47]. Families' behaviours or attitudes reframing or appraising the stress/situation in our research are mainly rooted in Confucian introspection, forbearance, and perseverance, but not religion [45]. Familism replaced the function of 
religion in Chinese families and weakened the relationship between the subscale of acquiring relatives' support and the subscale of seeking spiritual support [15]. Finally, the coping behaviours described in item 2 and item 16, item 3 and item 7, item 4 and item 20, item 6 and item 10, item 7 and item 22, and item 8 and item 10 had similar meaning or were correlated with each other as Figure 2 shows.

\section{Convergent validity}

The F-COPES had a satisfactory convergent validity. The correlation coefficient for the convergent validity of F-COPES was strong (the correlation coefficient between F-COPES and MSPSS $=0.50, P<0.01$ ). Through seeing how closely the new scale is related to other measures to which it should be related, this result confirmed that the Chinese version of F-COPES could measure family coping well [29]. The correlation of the subscale of seeking spiritual support with MSPSS was not significant because only $6.5 \%$ of family members in the current study had religious faith. When they are searching for help, spiritual support is not the common choice [15].

\section{Internal consistency reliability}

The Chinese version of F-COPES had perfect internal consistency as the Cronbach's alpha coefficient of this measure was 0.86 . The internal consistency reliability of the subscales were good as well, except for the passive appraisal subscale. This finding was similar to that in another study [13]. There are two possible explanations for this result. First, this result could be due to the population it measured. The passive appraisal is always the least used coping method among the families of patients with dementia [48]. In the current study, more than half of the family members only used one or two of these four coping methods, thereby influencing the internal consistency. Secondly, it might be associated with the fact that there were only four items in this subscale [49]. Taken together, our findings suggested we should add more items in this subscale focusing on the family members of patients with dementia to improve its internal consistency reliability by increased applicability and appropriate item redundancy.

\section{Strength and limitations}

The major strength of this study was that we developed the Chinese version of F-COPES and evaluated the psychometric of it in families of patients with dementia, including content validity, item homogeneity, factor structure, convergent validity, and internal consistency reliability of the F-COPES. Our research also has limitations. First, some items should be added, especially in the subscale of passive appraisal. Second, we urge caution in generalizing our findings to other populations since the study population was restricted to families of patients with dementia; future studies among other populations are warranted to confirm our findings.

\section{Conclusion}


This study demonstrated that the Chinese version of the F-COPES showed satisfactory psychometric properties. Our findings suggest that it may serve as a useful scale for assessing the coping behaviours in families of patients with dementia from the perspective of the family systems in China.

\section{Abbreviations}

F-COPES: Family Crisis Oriented Personal Evaluation Scales; MSPSS: Multidimensional Scale of Perceived Social Support; $\chi^{2} / \mathrm{df}$ : chi-square of model fit/df; CFI: comparative goodness-of-fit index; GFI: goodness-of-fit index; TLI: Tucker-Lewis Index; PGFI: parsimony goodness-of-fit index; RMSEA: root mean square error of approximation.

IQR: interquartile range; I-CVI: item content validity index; S-CVI: scale content validity.

\section{Declarations}

\section{Ethics approval and consent to participate}

The Ethical Review Committee of School of Nursing, Peking Union Medical College approved the study protocol in April 15 (Approval no. [2015] 03). All of the participants were informed about the aim and significance of this study, their right to quit at any time, and how to fill in the scales. All participants provided written informed consent.

\section{Consent for publication}

Not applicable.

\section{Competing interests}

The authors declared no potential conflicts of interest with respect to the research, authorship, and/or publication of this article.

\section{Funding}

The authors received financial support from Natural Science Foundation of Jiangsu Province (BK20201016) and Higher Education Philosophy and Social Science Foundation of Jiangsu Province (2019SJA0944). The funders had not directly role in the design, data collection, analysis, interpretation or writing of the manuscript.

\section{Authors' contributions}

QW was involved in the conception and design, acquisition of data, analysis, and interpretation of data, drafting and revising manuscript; SC and WL participated drafting and revising of the manuscript; YS contributed to the design, planning, coordination, and revision of manuscript. All authors have read and approved the manuscript. 


\section{Acknowledgment}

Not applicable.

\section{Availability of data and materials}

The datasets during and/or analysed during the current study available from the corresponding author on reasonable request.

\section{References}

1. American Psychiatric Association: The diagnostic and statistical manual of mental disorders (DSM5). Washington, DC: American Psychiatric Publishing; 2013.

2. Dementia [http://www.who.int/news-room/facts-in-pictures/detail/dementia]

3. Caregiving for Person with Alzheimer's Disease or a Related Dementia [https://www.cdc.gov/aging/caregiving/alzheimer.htm]

4. Ransmayr G, Hermann P, Sallinger K, Benke T, Seiler S, Dal-Bianco P, Marksteiner J, Defrancesco M, Sanin G, Struhal W et al. Caregiving and Caregiver Burden in Dementia Home Care: Results from the Prospective Dementia Registry (PRODEM) of the Austrian Alzheimer Society. J Alzheimers Dis. 2018; 63(1):103-14.

5. Maguire KC: Stress and Coping in Families. Cambridge: Polity Press; 2012.

6. Villarejo Galende A, Eimil Ortiz M, Llamas Velasco S, Llanero Luque M, Lopez de Silanes de Miguel C, Prieto Jurczynska C. Report by the Spanish Foundation of the Brain on the social impact of Alzheimer disease and other types of dementia. Neurologia. 2021;36(1):39-49.

7. McCubbin HI, Thompson A, McCubbin MA: Family measures: Stress, coping and resiliencyinventories for research and Practice. Honolulu: Kamehameha Schools 2001.

8. Daignault IV, Hebert M, Cyr M, Pelletier M, McDuff P. Correlates and predictors of mothers' adaptation and trauma symptoms following the unveiling of the sexual abuse of their child. $J$ Interpers Violence. in press.

9. Shapira R, Turbitt E, Erby LH, Biesecker BB, Klein WMP, Hooker GW. Adaptation of couples living with a high risk of breast/ovarian cancer and the association with risk-reducing surgery. Fam Cancer. 2018; 17(4):485-93.

10. Hassani P, Abbasi P, Tafreshi MZ, Zayeri F, Ziapour A. Persian version of Family Crisis Oriented Personal Evaluation Scales: psychometric properties. Int J Pediatr. 2018; 6(1):6919-30.

11. Alwi N, Harun D, Omar B, Ahmad M, Zagan M, Leonard JH. The internal reliability of Family Crisis Oriented Personal Evaluation Scale (F-COPES) in Malay version among caregivers of individual with learning disabilities. Clin Ter. 2015; 166(6):e361-e4. 
12. Sari A, Cetinkaya Duman Z. Strategies used by families to cope with chronic mental illnesses: psychometric properties of the family crisis oriented personal evaluation scale. Perspect Psychiatr Care. 2020; 56(2):462-73.

13. Lim J-w, Townsend A. Cross-ethnicity measurement equivalence of family coping for breast cancer survivors. Res Soc Work Pract. 2012; 22(6):689-703.

14. Chui WY, Chan SW. Stress and coping of Hong Kong Chinese family members during a critical illness. J Clin Nurs. 2007; 16(2):372-81.

15. Liang S: Outline of Chinese culture. Shanghai: Shanghai People's Publishing House; 2005.

16. Kim K. The relation among fit indexes, power, and sample size in structural equation modeling. Struct Equ Modeling. 2005; 12:368-90.

17. Coyne E, Dieperink KB, Ostergaard B, Creedy DK. Strengths and resources used by Australian and Danish adult patients and their family caregivers during treatment for cancer. Eur $\mathrm{J}$ Oncol Nurs. 2017; 29:53-9.

18. Ahlert IA, Greeff AP. Resilience factors associated with adaptation in families with deaf and hard of hearing children. Am Ann Deaf. 2012; 157(4):391-404.

19. Tay KC, Seow CC, Xiao C, Lee HM, Chiu HF, Chan SW. Structured interviews examining the burden, coping, self-efficacy, and quality of life among family caregivers of persons with dementia in Singapore. Dementia (London). 2016; 15(2):204-20.

20. Son H, Friedmann E, Thomas SA, Son YJ. Biopsychosocial predictors of coping strategies of patients postmyocardial infarction. Int J Nurs Pract. 2016; 22(5):493-502.

21. Zimet GD, Powell SS, Farley GK, Werkman S, Berkoff KA. Psychometric characteristics of the Multidimensional Scale of Perceived Social Support. J Pers Assess. 1990; 55(3-4):610-7.

22. Sousa VD, Rojjanasrirat W. Translation, adaptation and validation of instruments or scales for use in cross-cultural health care research: a clear and user-friendly guideline. J Eval Clin Pract. 2011; 17(2):268-74.

23. Yuan S, Wang F, Li X, Jia M, Tian M. Facilitators and barriers to implement the family doctor contracting services in China: findings from a qualitative study. BMJ Open. 2019; 9(10):e032444.

24. Dai B, Mao Z, Wu B, Mei YJ, Levkoff S, Wang H. Family caregiver's perception of Alzheimer's disease and caregiving in Chinese culture. Soc Work Public Health. 2015; 30(2):185-96.

25. Li X, Fang W, Su N, Liu Y, Xiao S, Xiao Z. Survey in Shanghai communities: the public awareness of and attitude towards dementia. Psychogeriatrics. 2011;11(2):83-9.

26. Li L: Chinese General Social Survey. In., 2020-10-1 edn. Beijing; 2017.

27. Lynn MR. Determination and quantification of content validity. Nurs Res. 1986; 35(6):382-5.

28. Polit DF, Beck CT: Nursing research: principles and methods, 7th edn. Philadelphia: Lippincott, Williams, \& Wilkins; 2004.

29. Streiner D, Norman G: Health measurement scales: a practical guide to their development and use. New York: Oxford University Press; 2008. 
30. Carmines EG, Mclver JP: Analyzing models with observable variables. In: Social measurement: Current issues. Edited by Borgatta EF, Bohrnstedt GW. Beverly Hills: Sage; 1981.

31. Bentler PM. Comparative fit indexes in structural models. Psychol Bull. 1990; 107(2):238-46.

32. Tucker LR, Lewis C. Reliability coefficient for maximum likelihood factor analysis. Psychometrika. 1973; 38(1):1-10.

33. Joreskog KG, Sorbom D: LISREL 8: User's reference guide. Chicago: Scientific Software International; 1996.

34. Bentler PM, Bonett DG. Significant tests and goodness of fit in the analysis of covariance structure. Psychol Bull. 1980; 88:588-606.

35. Brown MW, Cudeck R: Alternative ways of assessing model fit. In: Testing structural equation models. Edited by Bollen KA, Long JS. Newbury Park, CA: SAGE Publications; 1993: 136-62.

36. MacCallum R, Roznowski M, Necowitz LB. Model modifications in covariance structure analysis: the problem of capitalization on chance. Psychol Bull. 1992; 111 3:490-504.

37. Cheung GW-K, Rensvold RB. Evaluating goodness-of-fit indexes for testing measurement invariance. Struct Equ Modeling. 2002; 9(2):233-55.

38. Pihet S, Kipfer S. Coping with dementia caregiving: a mixed-methods study on feasibility and benefits of a psycho-educative group program. BMC Geriatr. 2018; 18(1):209.

39. Khamis H. Measures of Association: How to Choose? J Diagn Med Sonogr. 2008; 24(3):155-62.

40. DeVellis RF: Scale development theory and application. London: SAGE; 1991.

41. Zimet GD, Dahlem NW, Zimet SG, Farley GK. The Multidimensional Scale of Perceived Social Support. J Pers Assess. 1988; 52(1):30-41.

42. Kim SM, Han DH, Trksak GH, Lee YS. Gender differences in adolescent coping behaviors and suicidal ideation: findings from a sample of 73,238 adolescents. Anxiety Stress Coping. 2014; 27(4):439-54.

43. Youngblut JM, Brooten D. What children wished they had/had not done and their coping in the first thirteen months after their sibling's neonatal/pediatric intensive care unit/emergency department death. J Palliat Med. 2020; 24(2):226-32.

44. Guada J. The unique coping strategies of African American families with a loved one with schizophrenia: the Use of the Family Crisis Oriented Personal Evaluation Scale. J Fam Soc Work. 2012; 15(3):171-86.

45. Wang X, Shi M. A study of Chinese stress coping: Emic and etic approach. Adv in Psych Sci. 2013; 21(7):1239-47.

46. Wang Q, Sheng Y, Wu F, Zhang Y, Xu X. Effect of different sources support on adaptation in families of patient with moderate-to-severe dementia in China. Am J Alzheimers Dis Other Demen. 2019; 34(6):361-75.

47. Rush CL, Lobo T, Serrano A, Blasini M, Campos C, Graves KD. Complementary and alternative medicine use and Latina breast cancer survivors' symptoms and functioning. Healthcare (Basel). 2016; 4(4):80. 
48. Wilks SE, Little KG, Gough HR, Spurlock WJ. Alzheimer's aggression: influences on caregiver coping and resilience. J Gerontol Soc Work. 2011; 54(3):260-75.

49. Waltz CF, Strickland OL, Lenz ER: Measurement in nursing and health research, vol. 4. New York: Springer Publishing Company; 2010.

\section{Tables}

Table 1 Characteristics of patients and families $(\mathrm{N}=215)$ 


\begin{tabular}{|c|c|}
\hline Characteristics & Median (IQR)/\% \\
\hline \multicolumn{2}{|l|}{ Demographic and disease-related characteristics of patients } \\
\hline Age, median (IQR), years & $82.0(76.0-86.0)$ \\
\hline \multicolumn{2}{|l|}{ Gender } \\
\hline Female, \% & 65.1 \\
\hline Male, \% & 34.9 \\
\hline \multicolumn{2}{|l|}{ Subtypes of dementia } \\
\hline Alzheimer's disease, \% & 57.2 \\
\hline Vascular dementia, \% & 29.8 \\
\hline Others or unknown, \% & 13.0 \\
\hline \multicolumn{2}{|l|}{ Dementia severity } \\
\hline Moderate, \% & 51.2 \\
\hline Severe, \% & 48.8 \\
\hline Time or duration since diagnosis, median (IQR), months ${ }^{\text {a }}$ & $17.5(7.0-39.0)$ \\
\hline \multicolumn{2}{|l|}{ Demographic of the family members and the whole family } \\
\hline Age, median (IQR), years & $59.5(53.3-65.0)$ \\
\hline \multicolumn{2}{|l|}{ Education level ${ }^{b}$} \\
\hline Junior middle school and below, \% & 5.1 \\
\hline High school, \% & 33.5 \\
\hline College degree, \% & 51.6 \\
\hline Graduate degree, \% & 8.8 \\
\hline Having religious faith (yes) ${ }^{c}, \%$ & 6.5 \\
\hline Family income per month per person, median (IQR), Chinese Yuan ${ }^{d}$ & $4000.0(3500.0-8000.0)$ \\
\hline
\end{tabular}

Note. a: There were seven missing data; b: two missing data; $c$ : one missing data; d: 15 missing data. Continuous variables are expressed as median (interquartile range, IQR), and categorical variables are expressed as percentages 
Table 2 Item homogeneity of each item and the whole scale of the original version of the F-COPES $(\mathrm{N}=\mathbf{2 1 5})$

Note. a. If Cronbach's alpha coefficient of the subscale after deleting any item is higher than before, it means that the item has low homogeneity to other items.

b. Since corrected item-total correlation of item 12 and item 28 in the original subscale of passive appraisal were negative ( -0.26 and -0.50 , respectively), the scoring method of item 12 and item 28 were reversed, and the corrected item-total correlation, corrected item-subscale correlation and Cronbach's alpha coefficient of the subscale after deleting each item of the modified subscale of passive appraisal were increased.

Table 3 Fit statistics for original factor structure model of the Family Crisis Oriented Personal Evaluation Scales and the competing model in the present study $(\mathrm{N}=215)$

\begin{tabular}{lcccccl} 
Models & $\chi^{2} / \mathrm{df}$ & CFI & GFI & TLI & PGFI & RMSEA \\
\hline Original model $^{\mathrm{a}}$ & 1.97 & $\mathbf{0 . 8 6}$ & 0.83 & 0.85 & 0.68 & 0.07 \\
\hline Competing model $^{\mathrm{b}}$ & 1.65 & $\mathbf{0 . 9 1}$ & 0.85 & 0.90 & 0.69 & 0.06
\end{tabular}

Note. $\chi^{2} / \mathrm{df}=$ chi-square of model fit/df; CFI = comparative goodness-of-fit index; GFI = goodness-of-fit index; TLI = Tucker-Lewis Index; PGFI = parsimony goodness-of-fit index; RMSEA = root mean square error of approximation.

The following criteria were used to determine whether the models fit the data, including 3.00 or lower on the $\chi^{2}$ /df; 0.90 or greater on the CFI, GFI, and TLI; 0.50 or greater on PGFI; and an RMSEA of 0.08 or lower. The model that meets the above-mentioned fitness criteria and the value of $\mathrm{CFI}$ of the model is higher than the another model $(\triangle \mathrm{CFI} \otimes 0.01)$, indicates it is significantly better than the another model.

a. Original model was established based on the factor structure of the English version of the Family Crisis Oriented Personal Evaluation Scales.

b. Competing model was established based on parts of the original model and the classification of social support. In the competing model, we combined the three items in the original subscale of mobilizing the family to acquire and accept help, and the nine items in the original subscale of acquiring social support, and then redivided these 12 items into three subscales depending on the source of the social support, including the subscale of acquiring relatives' support, the subscale of acquiring friends' support, and the subscale of acquiring neighbours' or others' support.

Table 4 Convergent validity of the Chinese version of the Family Crisis Oriented Personal Evaluation Scales 
No. Items

Corrected item-total correlation
Corrected

item-

subscale

correlation
Cronbach's alpha coefficient of the subscale/

Cronbach's alpha coefficient of the subscale after deleting each item ${ }^{a}$

\section{Acquiring social support}

1 Sharing our difficulties with relatives

2 Seeking encouragement and support from friends

$5 \quad$ Seeking advice from relatives (grandparents, etc.)

8 Receiving gifts and favours from neighbours (e.g., taking in mail, etc.)

10 Asking neighbours for favours and assistance

16 Sharing concerns with close friends

18 Exercising with friends to stay fit and reduce tension

20 Doing things with relatives (gettogethers, dinners, etc.)

25 Asking relatives how they feel about problems we face

\section{Reframing}

3 Knowing we have the power to solve major problems

$7 \quad$ Knowing that we have the strength within our own family to solve our problems

11 Facing the problems "head-on" and trying to get a solution right away

13 Showing that we are strong

0.48

0.53

0.59

$0.55 \quad 0.64$

0.54

0.57

0.82

0.84
0.42
0.53
0.83

0.28

0.58

0.82

0.56

0.55

0.83

0.57

0.58

0.82

0.45

0.46

0.83

0.57

0.62

0.82

0.45

0.47

0.83

0.64

0.82
0.49
0.64
0.82

0.85

$\begin{array}{lllll}15 & \begin{array}{l}\text { Accepting stressful events as a fact of } \\ \text { life }\end{array} & 0.24 & 0.46 & 0.84\end{array}$

19 Accepting that difficulties occur unexpectedly

22 Believing we can handle our own

Believing we can handle our own
problems

$\begin{array}{lll}0.36 & 0.34 & 0.86\end{array}$

0.60

0.74

0.81 
24 Defining the family problem in a more

0.42

0.54

0.83

positive way so that we do not become

too discouraged

Seeking spiritual support

0.90

14 Attending worship or religious services

in religious places

23 Participating in religious activities

0.25

0.84

0.84

27 Seeking advice from a person in

0.19

0.78

0.88

religious places (such as a

minister, eminent monk, etc.)

\section{Mobilizing the family to acquire and accept help}

$4 \quad$ Seeking information and advice from

0.44

0.80

0.87

persons in other families who have

faced the same or similar problems

6 Seeking assistance from community agencies and programs designed to

help families in our situation

21 Seeking professional counselling and help for family difficulties
0.24
0.28
0.45

Original passive appraisal

0.06

12 Watching TV

$-0.26$

0.02

0.31

17 Knowing luck plays a big part in how well we are able to solve family

$0.07 \quad 0.09$

0.30

problems

26 Feeling that no matter what we do to prepare, we will have difficulty handling problems

28 Believing if we wait long enough, the problem will go away

\section{Modified passive appraisal ${ }^{b}$}

$\begin{array}{lll}0.12 & -0.01 & 0.20\end{array}$

0.50

$\begin{array}{lll}0.24 & 0.31 & 0.40\end{array}$

Watching TV

0.27

0.14

0.31

17 Knowing luck plays a big part in how well we are able to solve family problems

26 Feeling that no matter what we do to prepare, we will have difficulty handling problems

28 Believing if we wait long enough, the

problem will go away

$\begin{array}{lll}0.07 & 0.14 & 0.30\end{array}$

$-0.50$

0.01

0.25

0.33

0.14

0.23

0.20

0.51

0.19

0.25 
Subscales of Family Crisis Oriented Personal Evaluation Scales

Acquiring relatives' support

Acquiring friends' support

Acquiring neighbours' or others' support

Reframing

Seeking spiritual support

Passive appraisal

Total score

Note. MSPSS $=$ Multidimensional Scale of Perceived Social Support.

$* p<0.05 ; * * p<0.01 ; * * * p<0.001$.

\section{Figures}




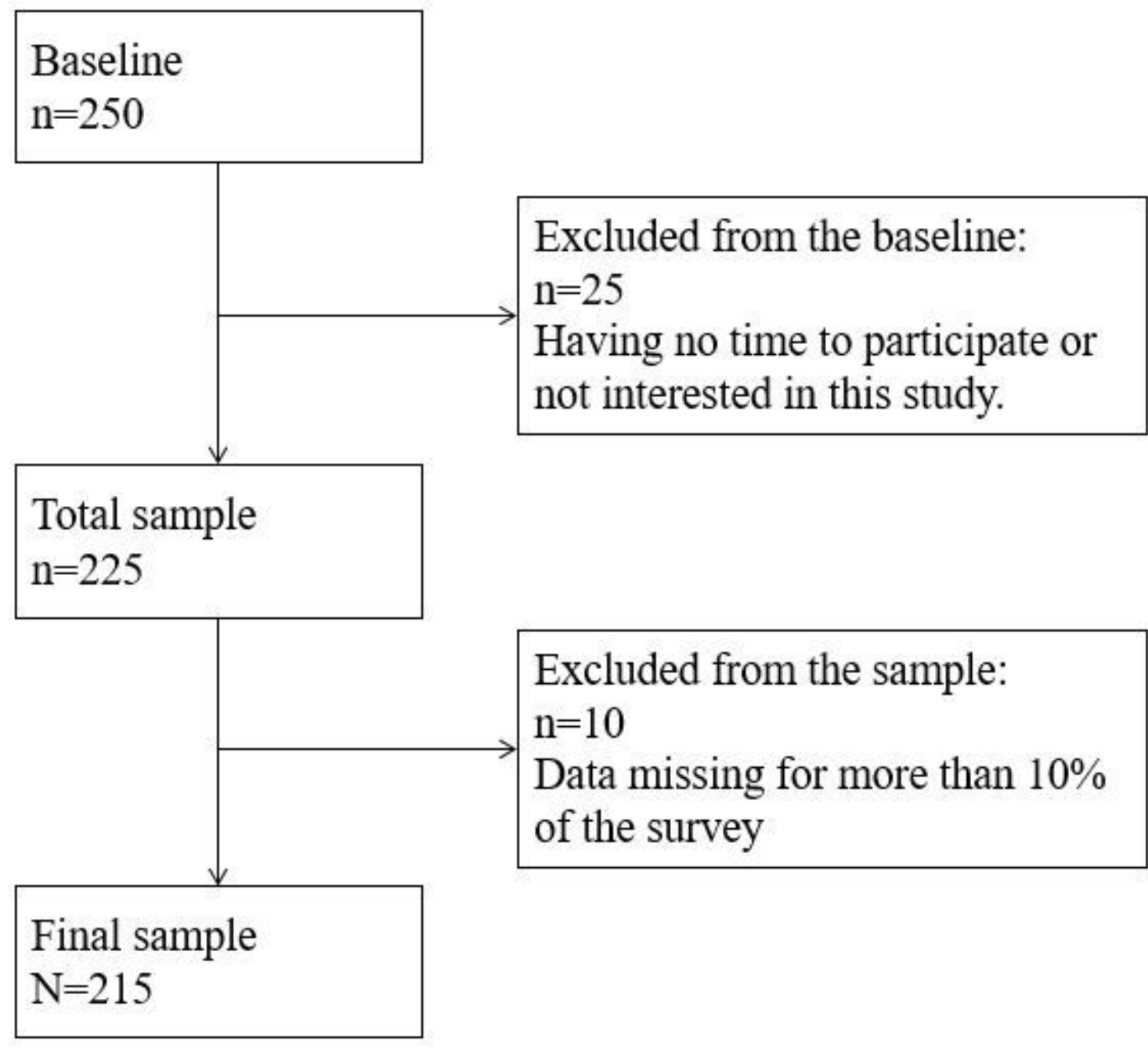

Figure 1

Assembly of the study sample 


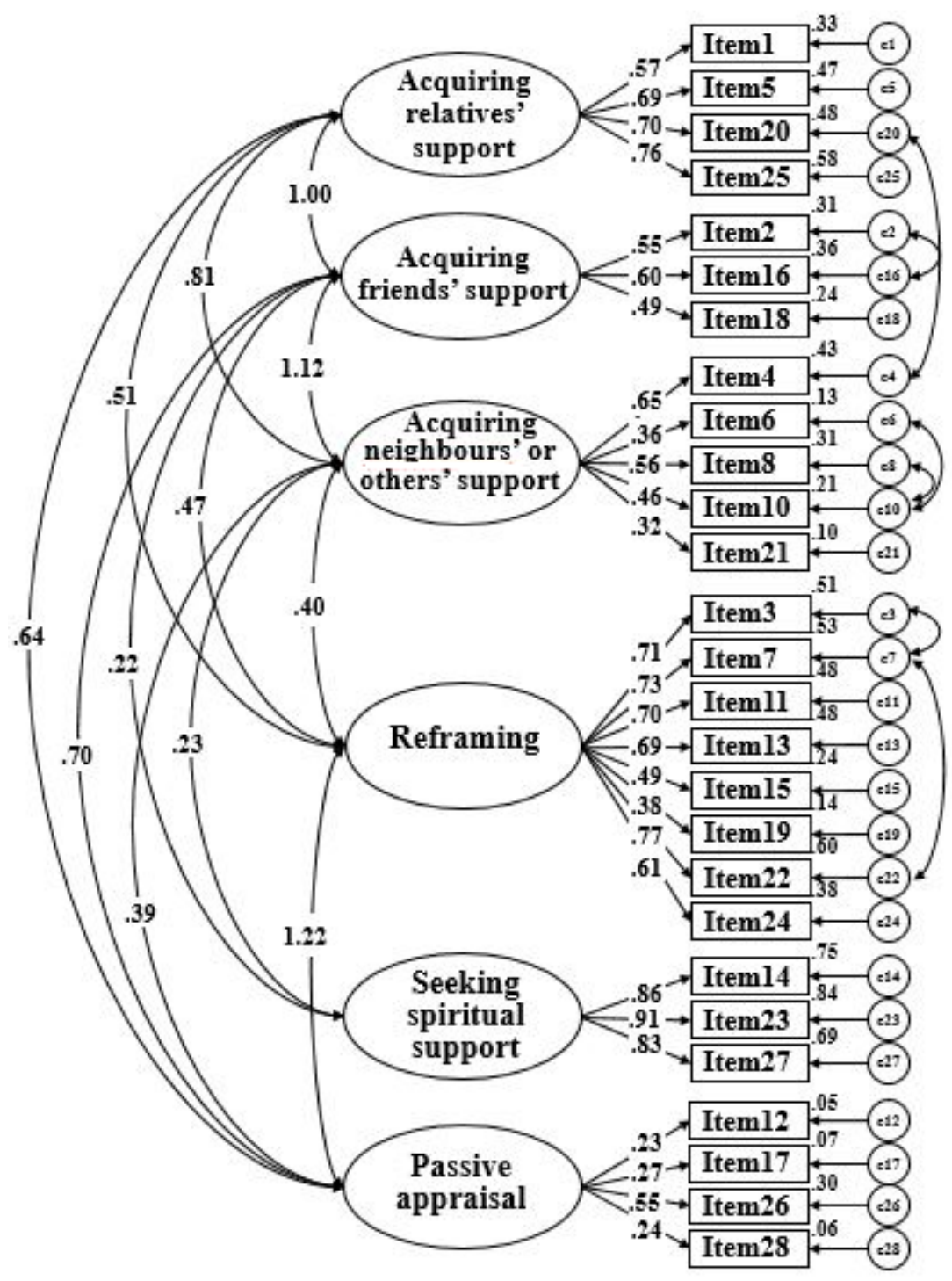

Figure 2

The factor structure of Chinese version of F-COPES 